\title{
The Morning After: Limit Violations and the Self-Regulation of Alcohol Consumption
}

\author{
Mark Muraven \\ University at Albany, State University of New York
}

\author{
R. Lorraine Collins and Elizabeth T. Morsheimer \\ State University of New York at Buffalo
}

\author{
Saul Shiffman and Jean A. Paty \\ University of Pittsburgh
}

\begin{abstract}
The authors examined whether the reciprocal relationship between alcohol consumption and distress unfolded over time in 2 samples of social drinkers. Participants monitored their alcohol intake and their cognitive and emotional responses to that drinking on hand-held computers. On mornings after drinking, those who had violated their self-imposed limits the day before reported more guilt, even after controlling for acute negative symptoms of drinking and amount consumed. Reciprocally, guilt led to poorer self-regulation of alcohol intake: Greater distress over alcohol consumption was linked to more intake, intoxication, and more limit violations. Individual differences moderated the relationships among limit violations, distress, and drinking. Consistent with the limit violation effect, violating a limit produced distress over consumption among social drinkers, and they responded to that distress by drinking more.
\end{abstract}

Keywords: abstinence violation effect, self-regulation, affect, ecological momentary assessment

Social drinkers often make efforts to set limits on their alcohol intake (Collins, Koutsky, Morsheimer, \& MacLean, 2001; Greenfield, Guydish, \& Temple, 1989). The reasons for limiting drinking may be situationally determined (e.g., driving or having a major test the next morning) or may be a stable individual difference (e.g., not wanting to feel out of control). Regardless of the reason, the self-regulatory processes underlying the adherence to limits are analogous to those involved in a variety of self-control tasks. Indeed, individuals' ability to limit their alcohol intake appears to be disrupted by the same processes that reduce self-control performance (Muraven, Collins, \& Nienhaus, 2002). Recent research has suggested that negative affective states can disrupt self-control (Tice, Bratslavsky, \& Baumeister, 2001), and therefore it is likely that negative affect may serve as a precursor to excessive and

Mark Muraven, Department of Psychology, University at Albany, State University of New York; R. Lorraine Collins and Elizabeth T. Morsheimer, Research Institute on Addictions, State University of New York at Buffalo; Saul Shiffman and Jean A. Paty, Department of Psychology, University of Pittsburgh.

Saul Shiffman and Jean A. Paty are founders and executive officers of Invivodata, Inc., which provides support for electronic diary methods in clinical trials. Portions of this research were presented at the 2002 Research Society on Alcoholism meeting in San Francisco and the 2003 Science of Real-Time Data Capture meeting in Charleston, SC. This research was supported by National Institute on Alcohol Abuse and Alcoholism Grants AA07595 and AA12770 awarded to R. Lorraine Collins and Mark Muraven, respectively. We thank the participants and the following persons who worked on the project: Charlene Vetter, Sandy Wilson, and Elizabeth Giles.

Correspondence concerning this article should be addressed to Mark Muraven, Department of Psychology, University at Albany, State University of New York, Albany, NY 12222. E-mail: muraven@albany.edu problem drinking (Cooper, Frone, Russell, \& Mudar, 1995; Marlatt \& Gordon, 1980, 1985). Moreover, this negative affect may be a product of self-control itself, as the result of violating a selfimposed limit (Collins, 1993; Heatherton, Polivy, \& Herman, 1990; Marlatt \& Gordon, 1980, 1985). That is, limit setting may be useful in regulating alcohol intake, but sometimes these limits can backfire and lead to more drinking, especially when the limit violation leads to negative affect.

\section{Reciprocal Relationship Between Emotions and Self-Control}

In a model based on Marlatt and Gordon's (1980, 1985) model of relapse prevention, Collins (1993) suggested that the violation of a drinking limit could bring about cognitive and emotional changes that generate subsequent drinking in social drinkers. This limit violation effect (LVE) occurs when individuals respond to a violation of their self-imposed limits on drinking by blaming themselves and experiencing guilt about the amount consumed. They then drink more alcohol to cope with the resultant negative affective state. In this way, there is a negative and reciprocal relationship between emotional distress and the self-regulation of alcohol intake.

According to this model, the limit violation, rather than excessive drinking per se or negative consequences related to excessive drinking (e.g., a hangover) is the critical factor in the experience of guilt and remorse. Failing at self-control, rather than the results of losing control, may be more likely to lead to distress (see, e.g., Norcross, Ratzin, \& Payne, 1989; Polivy \& Herman, 1999). This is especially likely to happen if the drinker attributes the limit violation to a personal weakness or failure. The model therefore predicts that there should be a relationship between violating personal drinking limits and distress. This relationship should be 
above and beyond any guilt produced by excessive drinking itself or acute negative effects related to drinking.

This cognitive-emotional reaction to a day's excessive drinking may, in fact, contribute to the misregulation of subsequent drinking. On the one hand, recognizing and regretting past excesses could promote more moderate drinking in the future. Negative physical consequences of excessive drinking also could serve as regulatory feedback by discouraging drinking the next day. On the other hand, research on drinking restraint (e.g., Bensley, Kuna, \& Steele, 1990; Ruderman \& McKirnan, 1984), some of which has examined the LVE (Collins \& Lapp, 1991; Collins, Lapp, \& Izzo, 1994), suggests that negative affective reactions over excess drinking may promote misregulation and further drinking. Distress triggered by violating a limit may produce more drinking, as the individual misregulates his or her emotions by drinking (see Baumeister, Heatherton, \& Tice, 1994; Tice et al., 2001). To test these opposing predictions, researchers need to investigate the relationships among recent drinking, cognitive and emotional responses to those specific drinking episodes, and subsequent drinking as they unfold over time.

Experimental investigations of components of the LVE (e.g., Bensley et al., 1990; Collins et al., 1994; Ruderman \& McKirnan, 1984) and similar models of self-regulation have produced some support for a reciprocal relationship between negative emotions and misregulation. However, the previous studies suffered from several weaknesses. First, the limit violations were induced artificially, which may have caused participants to make external rather than internal attributions for their loss of control. Second, because of the experimental nature of these studies, the induced limit violation might not have been of sufficient intensity to lead to subsequent increased drinking. Most of these studies also took place in the laboratory, with limited time frames, which could have interfered with the development or observation of the LVE. In addition, in most of these studies participants were not asked if they had violated their drinking limit; instead, a limit violation was presumed on the basis of other factors.

\section{Ecological Momentary Assessment}

The limitations of the experimental research illustrate the problem of investigating events that occur only occasionally in individuals' natural environments, develop over time, and that are easily biased in recall. Fortunately, new methods that permit the examination of behaviors as they occur in real time are helping researchers to avoid the problems associated with retrospective designs. Specifically, research using ecological momentary assessment (EMA) allows researchers to collect self-reports of mood, thoughts, and behaviors on an ongoing basis (Shiffman \& Stone, 1998). This ongoing assessment minimizes biases and maximizes the likelihood of capturing the nuances of events such as drinking. It also allows for collection of base rate information, which provides a context for understanding the target behavior.

In the present study, we attempted to avoid the limitations of previous research on the negative effects of emotions on selfregulation by using EMA methods to assess participants' drinking in their natural surroundings over time. We also obtained participants' self-report of limit violation, thereby ensuring that they actually had violated a limit on alcohol intake. By assessing how participants felt about their previous drinking and examining how those feelings relate to subsequent drinking, we can examine the reciprocal relationship between emotions and alcohol consumption, as it unfolds. Hence, the use of EMA methods allowed us to investigate the reciprocal relationship between negative affect and alcohol consumption in the daily lives of social drinkers.

In addition to illuminating the role of negative emotions in self-regulation, we were able to examine the influence of trait individual differences in the self-control of alcohol intake. In particular, heavier or problem drinkers may respond to a limit violation with greater distress, and that distress may be more harmful to their self-regulation of alcohol intake compared to light drinkers (Collins, 1993). Other individual-difference variables, such as individuals' temptation to drink and desire to regulate their alcohol intake, may also moderate the reciprocal relationship between alcohol consumption and distress. For example, individuals who are more concerned with maintaining their limits (high in restriction) may be more sensitive to a violation of their limits than individuals lower in restriction (Collins \& Lapp, 1991; Collins et al., 1994).

\section{The Present Studies}

Using EMA methods, we tested both the main effects of the relationship between violating a limit and self-regulation of alcohol intake and the moderating effects of individual differences. We tested this model in two separate and diverse populations, to increase the generalizability of the results. Study 1 consisted of a sample of underage (ages 18-20 years) drinkers. Study 2 consisted of a community sample of adult social drinkers (ages 21-45 years). After completing several individual-difference measures, both samples carried small hand-held computers that they used to enter self-reports of drinking immediately after a drinking episode. In addition to these self-initiated reports, the computer prompted participants to complete an assessment every morning. This morning assessment included questions about the previous day's drinking and participants' thoughts and feelings about their previous day's alcohol consumption (feeling bad about amount consumed, or guilt) and plans for future consumption.

As outlined above, negative affect related to alcohol consumption should follow from violating one's self-imposed drinking limits. The mere act of violating a limit on alcohol consumption may put the individual at risk for remorse, guilt, and other negative affective states. However, the LVE model predicts that violating one's self-imposed limit on consumption is the critical factor in the experience of guilt after drinking. In particular, experiencing a limit violation should produce distress, above and beyond any distress produced by the excessive drinking itself or the suffering of negative symptoms due to drinking (i.e., being hung over). The effects of a limit violation on subsequent distress may be stronger for individuals who are highly motivated to maintain their limits (high in trait restriction). Similarly, the effects may be stronger for individuals who are heavier drinkers, as the reciprocal cycle of the LVE may help explain the poorer self-regulation of alcohol intake among heavy drinkers.

We also predicted, on the basis of the LVE model, that experiencing a limit violation and feeling bad about that violation should be positively related to subsequent alcohol consumption ("I feel bad about how much I drank, so I will drink more to cope with those feelings"). In contrast to the LVE, one might predict that 
feeling bad about the amount consumed might help individuals regulate their alcohol intake ("I feel bad about how much I drank, so I will drink less to prevent those feelings from reoccurring"). In that case, there should be a negative relationship between distress over drinking and intention to drink that day. The present data will allow us to compare these two models (guilt as helpful vs. guilt as harmful) so that we can better understand the relationship between emotions and the regulation of alcohol intake. Individual differences, especially average weekly drinking, may moderate these relationships as well.

\section{Study 1}

\section{Method}

\section{Participants}

Study 1 consisted of 106 (49 male and 57 female) underage (mean age $=$ 19.3 years) social drinkers. Fliers, newspaper advertisements, and word of mouth were used to advertise this experiment. Interested persons called a dedicated telephone number, and a trained interviewer screened them for eligibility. In particular, participants had to be between the ages of 18 and 20 years, drink a minimum of four drinks a week $(M=18.6, S D=10.6)$ and have no previous medical diagnosis or treatment for substance abuse. In addition, they had to score 2 or less on the Short Michigan Alcohol Screening Test (Selzer, Vinokur, \& Rooijen, 1975). In short, this was a sample of underage social drinkers with no indication of alcohol problems or misuse.

\section{Procedure}

Participants first completed a series of questionnaires in small groups. At the end of that session, they indicated their interest and availability to participate in the second phase of the project. These participants were invited to return to the Research Institute on Addictions in Buffalo, New York, for training on the hand-held computer. At this session, they were given $1 \frac{1}{2}$ to $2 \mathrm{hr}$ of individualized training in the use of the small hand-held computer, the electronic diary (ED) used for self-monitoring behavior.

\section{Electronic Diary}

Participants in Study 1 carried a small hand-held computer (Palm Pilot Professional; 4.7 in $[11.9 \mathrm{~cm}] \times 3.2$ in. $[8.1 \mathrm{~cm}] \times 0.7$ in. $[1.8 \mathrm{~cm}] 9.6 \mathrm{oz}$ [272.2 g]; Palm Inc., Milpitas, CA) that used software specifically developed for this project. In particular, participants answered questions in a yes-no format (which often prompted further questions), on an 11-point scale, or by selecting the actual number (e.g., number of drinks). Participants answered the questions using a stylus to select the best answer. Within an assessment, the ED software was designed to prevent missing data and out-of-range responses (see Shiffman, Paty, Gnys, Kassel, \& Hickcox, 1996, for a more detailed description).

Once an individual had mastered the procedures, he or she was provided with an ED for self-monitoring of ongoing behavior. Participants returned to the research site on a weekly basis for data uploading, changing ED batteries, and feedback on use of the ED. During the 2 to 3 weeks of involvement in the study, each participant took part in approximately four individualized sessions. These sessions focused on ED training, questionnaire administration (2-3 hrs), and weekly ED feedback sessions that each lasted for $1 \mathrm{hr}$.

Participants were told that we were interested in the day-to-day behavior of social drinkers. They were instructed to use the ED on an ongoing basis and to continue to drink in their typical fashion. They were trained to interact with the ED on multiple occasions each day. They initiated assessments on waking each morning (morning assessment) and at the start (begin-drinking assessment) and end (end-drinking assessment) of each episode of drinking. A drinking episode was individually defined on the basis of criteria such as a change in location or time. In addition to the interactions initiated by the participants, the ED prompted participants at random times during the day (with the constraint that no prompts were issued within an hour of a drinking episode) to complete a random assessment (approximately four per day). Participants were also prompted to complete an evening assessment, every day around 8:00 p.m.

Of particular interest in this study was the relationship among the end-of-drinking assessment the previous day, the morning assessment that day, and the end-of-drinking assessment that day. The end-of-drinking assessment asked participants whether they had violated their self-imposed limit for that episode ("Did you drink more than intended?" answered on an 11-point scale with endpoints of NO!! and YES!!'). In addition, participants reported the number of standard drinks they consumed during the episode and whether they were intoxicated (on the 11-point scale).

In the morning assessment, participants rated their current mood and intentions to drink ("Do you plan to drink tonight?") and consumption during the previous day (number of drinks). The morning assessment also queried participants for any negative effects associated with drinking (e.g., nausea, headache), using an 11-point scale with endpoints of NO!! and YES!! Distress related to alcohol consumption was measured in the morning assessment using two questions (whether participants felt "bad about amount consumed" and whether they felt "guilty about amount consumed," rated on the 11-point scale; these two questions were significantly correlated, $r=.92, p<.001$, and hence were combined to assess distress, combined items $\alpha=.92$ ). Also, participants in Study 1 were first asked whether they were likely to drink in the future. An affirmative response on that question then led to a rating of intention to drink on an 11-point scale.

\section{Questionnaires}

At the initial session, participants completed several measures that were designed to assess trait individual differences related to alcohol consumption. Two of these measures are relevant to the present study.

General information questionnaire. We used a general information questionnaire, which has been used in previous research (e.g., Collins et al., 1998), to assess demographic characteristics (e.g., gender, age, marital status), drinking-related information (e.g., typical weekly consumption, age at which one first tasted alcohol), typical experience of negative alcohol-related consequences (e.g., nausea, accidents while intoxicated), and typical use of drugs other than alcohol.

Temptation and Restraint Inventory. The Temptation and Restraint Inventory (TRI; Collins \& Lapp, 1992) is a 15-item measure of a participant's trait level of temptation to drink and concerns about regulating alcohol intake. It has five factors (Govern, Emotion, Cognitive Preoccupation, Concern About Drinking, and Restrict) with internal consistencies (alphas) ranging between .78 and .91. The five factors combine to form two internally consistent higher order factors: (a) Cognitive and Emotional Preoccupation (CEP, $\alpha=.79$ ) and (b) Cognitive and Behavioral Control (CBC, $\alpha=.91$ ). The CEP factor measures the temptation to drink, and the CBC factor measures concern about the control or regulation of alcohol intake (Collins, Koutsky, \& Izzo, 2000). Both higher order factors have been related to alcohol intake and also may be related to how individuals respond to limit violations (Collins \& Lapp, 1991; Collins et al., 1994).

\section{Overview of Data Analysis}

Previous research using data from the ED has focused on drinking episodes, with a particular interest in how amount consumed is related to mood and attributions for drinking immediately before and after the episode (Muraven, Collins, Morsheimer, Shiffman, \& Paty, 2005). In this study, we examined drinking at a more macro level: amount consumed during a given day. 
Our goal was to describe the within-subject relationship of the previous day's alcohol consumption and participants' morning-after reports of intoxication, violation of self-reported limits, acute negative effects due to drinking (e.g., hangovers), and feeling bad about amount consumed. We then examined the relationship among self-reported distress, limit violations, and drinking that day. We also assessed whether between-subject factors, such as age, trait temptation to drink, and gender, moderated the relationship between alcohol consumption and morning reports. Analyzing these data by aggregating across individuals and using standard regression equations would violate the assumption that the variance of the residuals is constant (Schwartz \& Stone, 1998). Moreover, standard regression would not permit the simultaneous analysis of between- and within-subject data. To avoid these problems, we treated the results as multilevel (days within participants).

We analyzed the data using Bryk, Raudenbush, and Congdon's (2000) hierarchical linear modeling (HLM) software using restricted maximum likelihood equations. Following previous recommendations (e.g., Kreft, de Leeuw, \& Aiken, 1995; Schwartz \& Stone, 1998), we person-centered all within-subject variables and grand mean centered all between-subject variables. For all analyses, an examination of residuals indicated that there were no significant outliers or influential cases.

\section{Results}

Participants in Study 1 carried the ED for an average of 21 days. During that time, they completed a total of 2,096 morning assessments and 947 after-drinking assessments. They drank alcohol on 7.37 days (range $=1-20, S D=3.55$ ) and on average consumed 5.67 drinks $(S D=4.23)$ on those days. The statistical power of HLM depends on both the number of observations (e.g., morning reports) and the number of groups (e.g., people). In particular, the number of observations is related to the estimation of the first level (within-subject) slopes (Kenny, Kashy, \& Bolger, 1998). Therefore, our power to detect within-subject relations was likely adequate.

On days after drinking, participants felt that they were moderately intoxicated the previous day $(M=5.56, S D=3.16$, one question, range $=1-11)$. They were slightly negative about the amount they consumed $(M=4.84, S D=3.49$, two questions, range $=2-22, \alpha=.92$ ), although their hangover symptoms (e.g., nausea, headache) were minor $(M=7.58, S D=1.00$, seven questions, range $=7-77, \alpha=.55)$. They indicated that they had intended to drink that night $31 \%$ of the time. Of those who indicated they were going to drink (only participants who answered the first question affirmatively were asked this question), they rated the intention as fairly strong $(M=8.49, S D=1.75$, one question, range $=1-11$ ).

The multilevel regressions on amount consumed and morning reports examined how the previous day's drinking affected participants' cognitions and feelings about alcohol in the morning and how those cognitions and feelings were related to subsequent drinking.

\section{Previous Day}

The LVE model predicts that the amount of alcohol participants consumed the previous day should be related to their cognitions and feelings about alcohol that morning. We controlled for day of week using six dummy codes (entered as fixed effects; these are not shown in the equations below to improve readability), as alcohol consumption and other behaviors may vary regularly over the week (Argeriou, 1975; West \& Hepworth, 1991). In particular, the following equations were specified in HLM:

$$
\begin{aligned}
& \text { Distress }_{i j}=b_{0 i}+b_{1 i}\left(\text { Previous day's limit violation }_{i j}\right)+e_{i j}(1) \\
& b_{0 i}=\gamma_{00}+\gamma_{01}\left(\mathrm{TRI}-\mathrm{CEP}_{i}\right)+\gamma_{02}\left(\mathrm{TRI}-\mathrm{CBC}_{i}\right) \\
& +\gamma_{03}\left(\text { Gender }_{i}\right)+\gamma_{04}(\text { Average weekly drinking } i)+u_{0 i} \\
& b_{1 i}=\gamma_{10}+\gamma_{11}\left(\mathrm{TRI}-\mathrm{CEP}_{i}\right)+\gamma_{12}\left(\mathrm{TRI}-\mathrm{CBC}_{i}\right) \\
& +\gamma_{13}\left(\text { Gender }_{i}\right)+\gamma_{14}(\text { Average weekly drinking } i)+u_{1 i} \text {. }
\end{aligned}
$$

Equation 1 is the within-subject effects (Level 1); that is, distress ${ }_{i j}$ is individual $i$ 's feelings of distress over alcohol consumption on day $\mathrm{j}, b_{\mathrm{O} i}$ is the average day's distress for each individual, and $b_{1 i}$ is the effects of previous day's limit violation on that morning's distress. The random error is captured by $e_{i j}$. In these equations, $b_{1 i}$ is the partial within-subject regression coefficients for each person $j$. The independent variables were person centered, and therefore the coefficients represent the increase in amount consumed for each unit increase in affect above the individual's average.

Two separate Level 2 equations were specified for each of the within-subject variables $\left(b_{0 i}\right.$ and $\left.b_{1 i}\right)$. These equations represent variation between participants. Equation 3 enabled us to model the effects of individual differences in TRI-CEP, TRI-CBC, gender, and average weekly drinking as well as the effects of random variation $\left(u_{2 i}\right)$ on the relationship between alcohol consumption and distress. To reduce collinearity among the Level 2 predictors, and to aid in the interpretation of main effects in the presence of interactions, the Level 2 predictors (individual differences) were grand mean centered.

The LVE predicts that guilt or remorse in regard to drinking should be produced by limit violations. Acute physical symptoms (hangovers) and excessive drinking also may lead to feelings of distress over alcohol consumption, but the effects of a limit violation should be above and beyond the effects of these variables on distress. As predicted by the LVE model, participants who reported violating their personal drinking limit during a drinking episode reported more distress over alcohol consumption the following morning $(B=1.28, S E=0.125), t(98)=10.2, p<.001$. Separate analyses also found that the more participants drank the night before, the more guilt they felt about how much they consumed $(B=0.651, S E=0.0405), t(98)=16.1, p<.0001$, and hangover symptoms also were related to feelings of distress $(B=$ 0.643, $S E=0.0282), t(98)=22.8, p<.0001$. When these variables are analyzed together, the effects of experiencing a limit violation the night before on distress the next morning remained significant even after controlling for the amount consumed and hangover symptoms $(B=0.129, S E=0.0389), t(97)=3.32, p<$ .001 . The results indicated that limit violations may be a critical factor in producing remorse, above and beyond the effects of excessive drinking or being hung over. Put another way, one beer can lead to considerable distress the next day, if one feels that a limit violation had occurred.

Several individual differences moderated the relationship between distress over a limit violation and amount consumed the previous day. As trait restriction (TRI-CBC) increased, the association between experiencing a limit violation and distress became stronger $(B=0.00714, S E=0.00374), t(98)=1.91, p<.05$. 
Limit violations lead to more guilt for individuals high in trait restriction, which is consistent with the restraint model (Collins \& Lapp, 1992). Trait temptation, as predicted, did not moderate the relationship between limit violations and guilt $(B=0.00133, S E=$ $0.00244), t(98)=0.543, n s$.

Participants' average weekly drinking also moderated the relationship between consumption and distress over alcohol consumption the next morning $(B=0.00494, S E=0.00271), t(98)=1.83$, $p<.07$. As shown on Figure 1, heavier drinkers are more sensitive to limit violations than lighter drinkers. The LVE model suggests that responses to guilt may help explain the progression from social to more problematic drinking. The finding that heavier drinkers respond to limit violations with greater distress is consistent with that model. Finally, there was an effect of gender in Study 1: Men were more sensitive to limit violations than women $(B=0.111, S E=0.0609), t(98)=1.82, p<.07$.

\section{Effects on Subsequent Drinking}

The LVE model predicts that drinking to excess may produce guilt and that guilt may lead to excessive drinking. Thus, there should be a relationship between distress over a limit violation and alcohol consumption that day. Subsequent consumption and selfreports of limit violation were calculated from participants' reports of drinking episodes on the ED. In the analyses, the amount participants consumed the previous day (to control for the individuals' general predisposition to drink) and the day of the week (dummy coded) were partialed out (the results were similar and significant when we did not control for previous day's consumption or day of week) using the following equations:

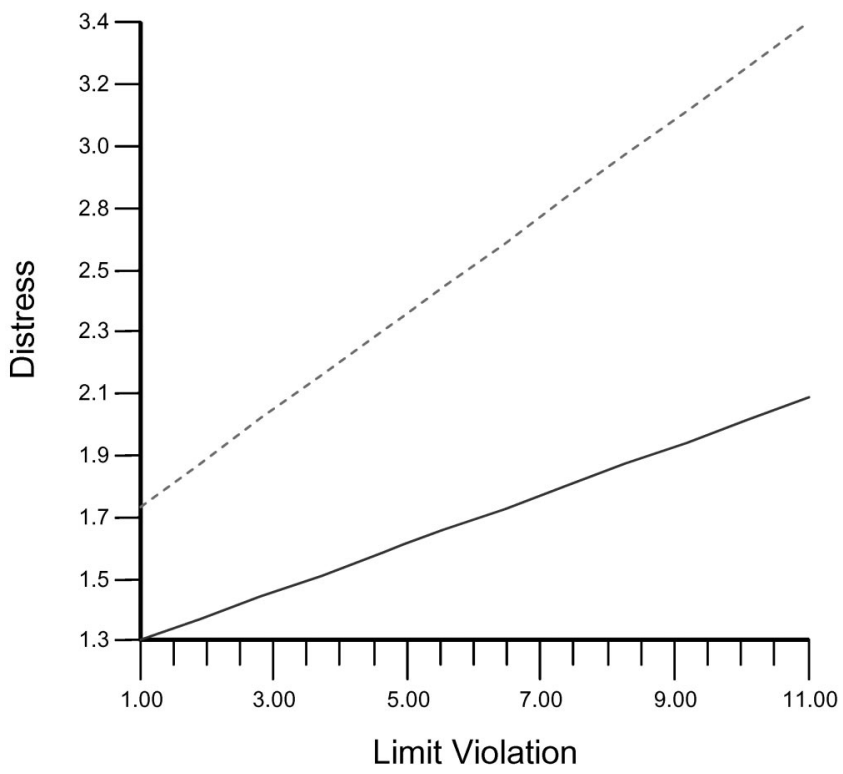

Figure 1. Relationship between experiencing a limit violation and distress, controlling for day of week, alcohol consumption, and hangover symptoms, in Study 1. Lines are plotted at one standard deviation above and below the sample mean of average weekly drinking. Stippled line $=$ heavy drinker; solid line $=$ light drinker.
Subsequent consumption ${ }_{i j}=b_{0 i}+b_{1 i}\left(\right.$ Distress $\left._{i j}\right)$

$$
\begin{gathered}
+b_{2 i}\left(\text { Previous day's consumption }_{i j}\right)+e_{i j} \\
b_{0 i}=\gamma_{00}+\gamma_{01}\left(\mathrm{TRI}_{-}-\mathrm{CEP}_{i}\right)+\gamma_{02}\left(\mathrm{TRI}-\mathrm{CBC}_{i}\right) \\
+\gamma_{03}\left(\text { Gender }_{i}\right)+\gamma_{04}\left(\text { Average weekly drinking }_{i}\right)+u_{0 i} \\
b_{1 i}=\gamma_{10}+\gamma_{11}\left(\mathrm{TRI}-\mathrm{CEP}_{i}\right)+\gamma_{12}\left(\mathrm{TRI}-\mathrm{CBC}_{i}\right) \\
+\gamma_{13}\left(\text { Gender }_{i}\right)+\gamma_{14}\left(\text { Average weekly drinking }_{i}\right)+u_{1 i} \\
b_{2 i}=\gamma_{20}+u_{2 i} .
\end{gathered}
$$

In Study 1, there was a direct relationship between distress over alcohol consumption and amount consumed based on the drinking episodes entered that day $(B=0.134, S E=0.0423), t(98)=3.14$, $p<.001$; that is, individuals who were more distressed by the amount they consumed the previous day drank more that day than individuals who were less distressed. This relationship held even after controlling for hangover symptoms (along with day of week and amount consumed the previous day) $(B=0.169, S E=$ $0.0517), t(97)=3.27, p<.001$. Distress also was associated with becoming intoxicated that day $(B=0.0924, S E=0.0307), t(98)=$ $3.01, p<.005$, and with violating a personal drinking limit while drinking $(B=0.0976, S E=0.0331), t(98)=2.95, p<.005$. Indeed, distress over alcohol consumption was related to more drinking the next day ( 2 days after the initial drinking episode, controlling for day of week and amount consumed in the past 2 days) $(B=0.124, S E=0.0351), t(97)=3.53, p<.001$. Regretting excessive drinking may lead to excessive drinking, intoxication, and limit violations that day and on subsequent days.

Finally, average weekly drinking moderated the relationship between distress over a limit violation and subsequent alcohol consumption $(B=0$. 0122, $S E=0.00215), t(98)=5.70, p<$ .0001 (see Figure 2). Heavier drinkers are more sensitive to the effects of guilt, just as they are more sensitive to the effects of violating their limits. Thus, the cycle of misregulation is stronger for heavier drinkers than for lighter drinkers. Gender also moderated the relationship between feeling bad about the amount consumed the previous day and amount consumed that day $(B=$ 0.149, $S E=0.0736), t(98)=2.02, p<.05$. Men were more sensitive to the effects of a limit violation than women, and they responded to distress by drinking more than women did. Trait restriction or temptation did not alter the relationship between distress and consumption.

\section{Test of Mediation}

Although statistical models of mediation in multilevel models are still being developed (Kenny, Korchmaros, \& Bolger, 2003; Krull \& MacKinnon, 1999), research suggests that a reformulization of the single-level test of mediation to the multilevel model is adequate for most samples. The LVE model suggests that the relationship between violating a personal limit and subsequent excessive drinking may be mediated by guilt. Consistent with that argument, the direct relationship between violating a limit to amount consumed the next day is reduced from $B=0.0961, S E=$ 0.0427, $t(98)=2.25, p<.025$, to $B=0.0652, S E=0.0403$, $t(97)=1.61, n s$, when guilt was included in the equation. The Goodman test of mediation confirmed that distress over drinking 


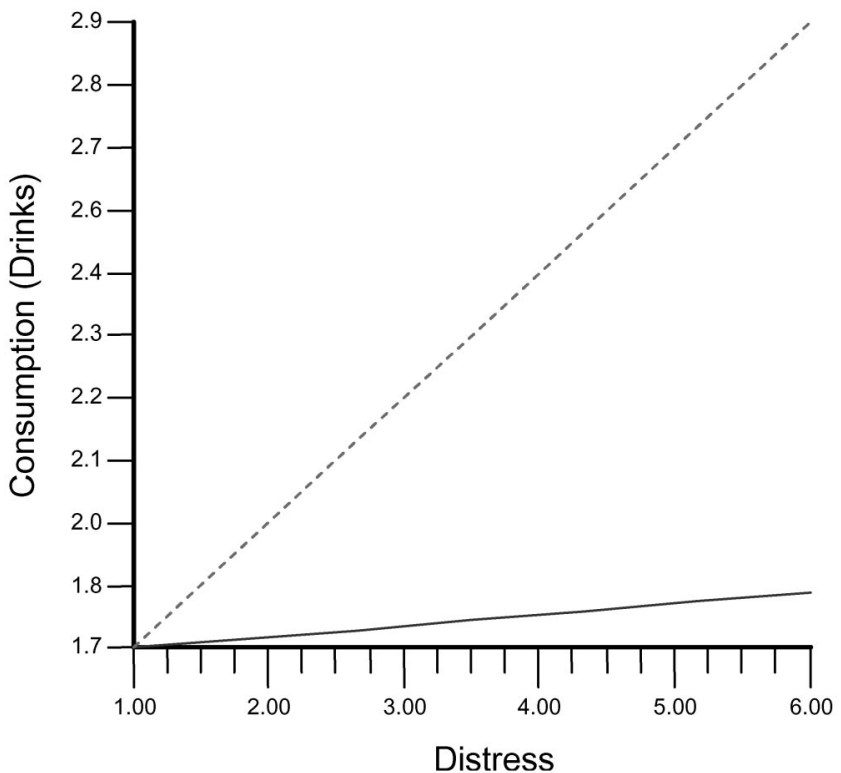

Figure 2. Relationship between self-reported distress in the morning and number of drinks consumed that day, controlling for day of week and previous day's alcohol consumption, in Study 1. Lines are plotted at one standard deviation above and below the sample mean of average weekly drinking. Stippled line $=$ heavy drinker; solid line $=$ light drinker.

was a significant mediator of the relationship between limit violation and subsequent drinking (Goodman test $=3.00, p<.005$; Baron \& Kenny, 1986; Kenny et al., 1998).

\section{Study 2}

The results of Study 1 strongly suggest that underage social drinkers may respond to a limit violation with negative affect and that this negative affect may lead to greater subsequent consumption. To replicate and extend these results, we tested the predictions of the LVE model in a very different sample of social drinkers. In particular, by examining the relationship among limit violations, negative affect, and alcohol consumption in a sample of adult social drinkers who have a longer history of drinking, different peers, and legal status, and who are in a different developmental period, we hope to improve the generalizability of our findings while lending further support to the model.

\section{Method}

\section{Participants}

The second sample consisted of 38 (20 male and 18 female) community residents who responded to newspaper advertisements for a study of daily drinking. They were social drinkers between the ages of 21 and 50 years $(M=26.9, S D=5.6)$ who drank a minimum four drinks a week $(M=$ $15.8, S D=10.9$ ) and who had no previous medical diagnosis or treatment for alcohol abuse. As in Study 1, participants were screened with the Short Michigan Alcohol Screening Test to ensure a sample of adult social drinkers who have no indication of problems with alcohol or alcoholism. Approximately $81.6 \%$ of the sample reported a European American background, and $26.3 \%$ were married.

\section{Procedure}

The procedures used in Study 2 were very similar to the methods used in Study 1. When participants in Study 2 were accepted into the study, they traveled to the Research Institute on Addictions for an initial, individual appointment. At that time, they completed a set of questionnaires (thus the questionnaire and training sessions were combined, unlike Study 1) and were given $1 \frac{1}{2}$ to $2 \mathrm{hr}$ of individualized training in the use of the small, hand-held computer, the ED used for self-monitoring behavior. As in Study 1 , participants completed the TRI and general information questionnaire at the training session.

\section{Electronic Diary}

Participants in Study 2 carried a small hand-held computer (PSION Organizer II LZ 64; 5.6 in. [14.2 cm] $\times 3.1$ in. $[7.9 \mathrm{~cm}] \times 1.1$ in. $[2.8 \mathrm{~cm}]$; $8.8 \mathrm{oz}$ [249.5 g]; PSION Ltd., London). Questions were presented in simple language on a four-line, 20 character LCD screen. Participants used arrow keys to scroll through alternative responses and pressed "Enter" to select the appropriate response. The ED interviews were mostly the same as in Study 1, with several notable differences. There was no evening assessment in Study 2, and ratings were made using a 4-point (instead of 11-point) Likert scale with anchors of NO!!, no, yes, and YES!!

More significantly, distress over a limit violation was assessed using one question ("feel bad about drinking?") in Study 2 (unlike the two questions in Study 1). Participants in Study 2 were asked to rate their likelihood of drinking in the future on a 4-point scale, without the gating question used in Study 1. Thus, intention to drink was measured very differently in the two samples. The after-drinking assessment in Study 2 included a measure of alcohol intake (standard drinks) but, unlike Study 1, it did not include measures of intoxication or limit violations. Instead, participants in Study 2 were asked in the morning interview whether they had violated their drinking limit the day before ("violate limit?"). Otherwise, the two studies are similar and the hypotheses were tested using similar methodology.

\section{Results}

Participants in Study 2 completed 539 morning assessments and 318 after-drinking assessments during the 15 days they carried the ED. They consumed alcohol on an average of 6.62 days (range $=$ $2-4, S D=3.18$ ) and averaged 5.85 drinks on days that they drank $(S D=2.89)$.

The morning assessments confirmed the accuracy of participants' reports of alcohol consumption during the previous day. They reported that their entries were correct $97.2 \%$ of the time. On mornings after drinking, participants felt that they were somewhat intoxicated the day before $(M=1.79, S D=1.05$, one question, range $=1-4)$. They typically did not feel much guilt or remorse $(M=1.18, S D=0.55$, one question, range $=1-4)$ and only felt like they slightly violated their drinking limit $(M=1.20, S D=$ 0.57 , one question, range $=1-4$ ). Finally, participants' reports of acute negative effects of alcohol (e.g., nausea, headache, blackout) were combined to create a single scale of negative effects associated with drinking. After drinking, participants in Study 2 were slightly hung over $(M=7.24, S D=2.32$, six questions, range $=$ $6-24, \alpha=.73)$. They rated their likelihood of drinking that night as moderately high $(M=2.39, S D=1.05$, one question, range $=$ $1-4)$.

\section{Effects of Previous Day's Consumption}

To test the LVE model, equations like those in Study 1 were specified in Study 2, with the addition of age to the Level 2 
equations. First, greater consumption the day before was associated with feeling that a violation of drinking limits had occurred $(B$ $=0.163, S E=0.0132), t(32)=12.5, p<.0001$. As predicted by the LVE model, remorse about alcohol consumption the next morning was related to the amount consumed the previous day $(B$ $=0.161, S E=0.0105), t(32)=15.4, p<.0001$. Controlling for physical symptoms of excessive drinking weakened but did not eliminate the relationship between distress and amount consumed the previous day $(B=0.021, S E=0.011), t(31)=1.85, p<.06$. Similarly, the relationship between amount consumed the previous day and limit violation remained after controlling for hangover symptoms $(B=0.021, S E=0.011), t(31)=1.87, p<.06$.

As in Study 1, there was a significant relationship between violating a drinking limit and feelings of guilt within the morning assessment $(B=0.960, S E=0.041), t(32)=23.6, p<.001$, after controlling for day of week. The addition of hangover symptoms and previous day's alcohol consumption weakened but did not completely eliminate the association between violation and distress $(B=0.361, S E=0.170), t(32)=2.12, p<.05$. As predicted by the LVE model, experiencing a limit violation was associated with feelings of distress, beyond that which could be explained by the alcohol consumption itself or negative physical symptoms.

Replicating Study 1, trait restriction (TRI-CBC) moderated the relationship between experiencing a limit violation and feeling bad about alcohol consumption $(B=0.0072, S E=0.00430), t(32)=$ $2.03, p<.05$. Individuals who were high in restriction were more sensitive to limit violations than individuals lower in trait restriction. Unlike Study 1, trait temptation also moderated this relationship $(B=-0.00572, S E=0.00162), t(32)=3.54, p<.01$. Individuals high in temptation were less responsive to a limit violation than individuals lower in temptation to drink. As shown in Figure 3, average weekly consumption also affected the rela-

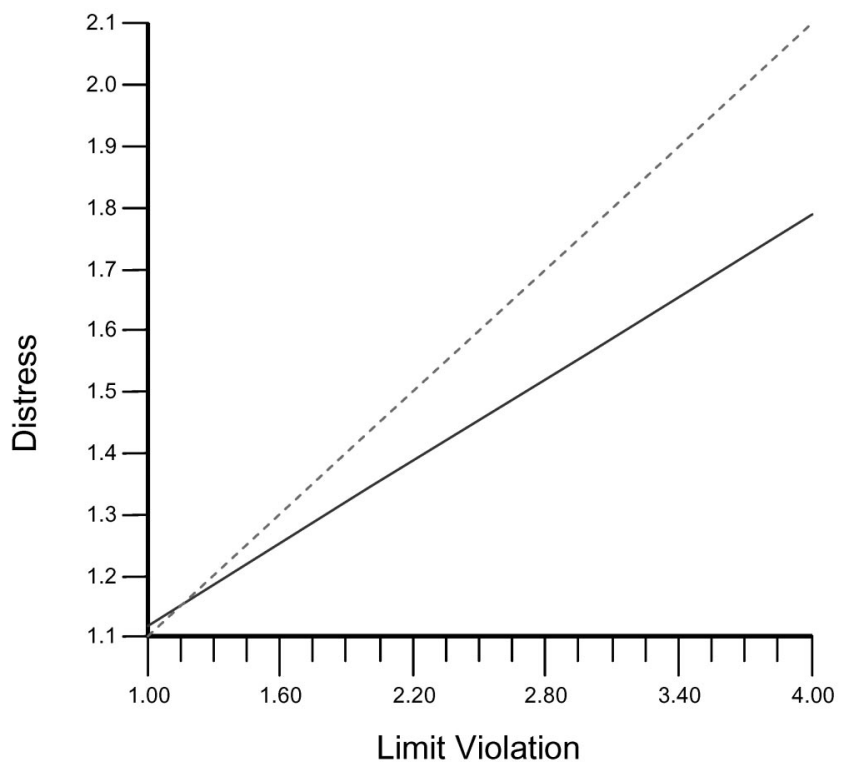

Figure 3. Relationship between experiencing a limit violation and distress, controlling for day of week, alcohol consumption, and hangover symptoms, in Study 2. Lines are plotted at one standard deviation above and below the sample mean of average weekly drinking. Stippled line $=$ heavy drinker; solid line $=$ light drinker. tionship between limit violations and distress $(B=0.00455, S E=$ $0.00208), t(32)=2.19, p<.05$. Heavy drinkers also were more sensitive to distress over a limit violation than lighter drinkers. This is consistent with the results found in Study 1 and consistent with the LVE model of guilt negatively influencing the selfregulation of alcohol intake. Finally, gender moderated the relationship $(B=0.103, S E=0.0488), t(32)=2.19, p<.05$. Men responded more strongly to limit violations than women. The association between limit violations and distress was not affected by participants' age, however $(B=0.00352, S E=0.00308)$, $t(32)=1.14, n s$.

\section{Effects on Subsequent Drinking}

In Study 2, the relationship between distress over a limit violation and consumption was mediated by intention to drink. Individuals who were more distressed the morning after drinking reported greater intentions to drink that day, after controlling for previous day's drinking and day of week $(B=0.277, S E=0.122), t(32)=$ $2.28, p<.05$. Similarly, participants' morning reports of violating their drinking limit was related to greater intention to drink that day $(B=0.233, S E=0.112), t(32)=2.07, p<.05$. Feeling bad or violating one's drinking limits increases the desire to drink that day, as predicted by the LVE model.

As would be expected, intentions to drink were strongly related to amount consumed on that day $(B=1.01, S E=0.145), t(32)=$ $6.98, p<.001$. Although distress over a limit violation was not directly associated with later day consumption, distress was associated with intentions to drink and intention to drink was associated with later day drinking.

As found in Study 1, between-subject individual differences moderated the relationship between feeling bad about the amount consumed the previous day and intention to drink that night. Individuals who drank more on average were more affected by distress than individuals who drank less (see Figure 4$)(B=0.221$, $S E=0.120), t(32)=1.84, p<.07$. The impact of distress over a limit violation was greater on heavy drinkers than on light drinkers. No other individual-difference variable, including age, gender, and trait temptation or restraint, moderated the relationship between distress and intention to drink that night.

\section{Test of Mediation}

Consistent with Study 1, the direct relationship between violating a limit to intention to drinking that following night was reduced from $B=0.281, S E=0.0986, t(32)=2.85, p<.01$, to $B=0.0478, S E=0.122, t(31)=0.391, n s$, when distress over drinking is included in the equation. Distress over drinking was a significant mediator of the relationship between limit violation and intention to drink (Goodman test $=2.25, p<.025)$.

\section{General Discussion}

We found that the failure to regulate alcohol intake involved a reciprocal, within-subject relationship between alcohol consumption and feelings of distress over a limit violation that unfolded over time. As predicted by the LVE model, how drinkers felt in the morning was significantly related to violating their self-imposed limit on alcohol consumption the day before. In particular, the 


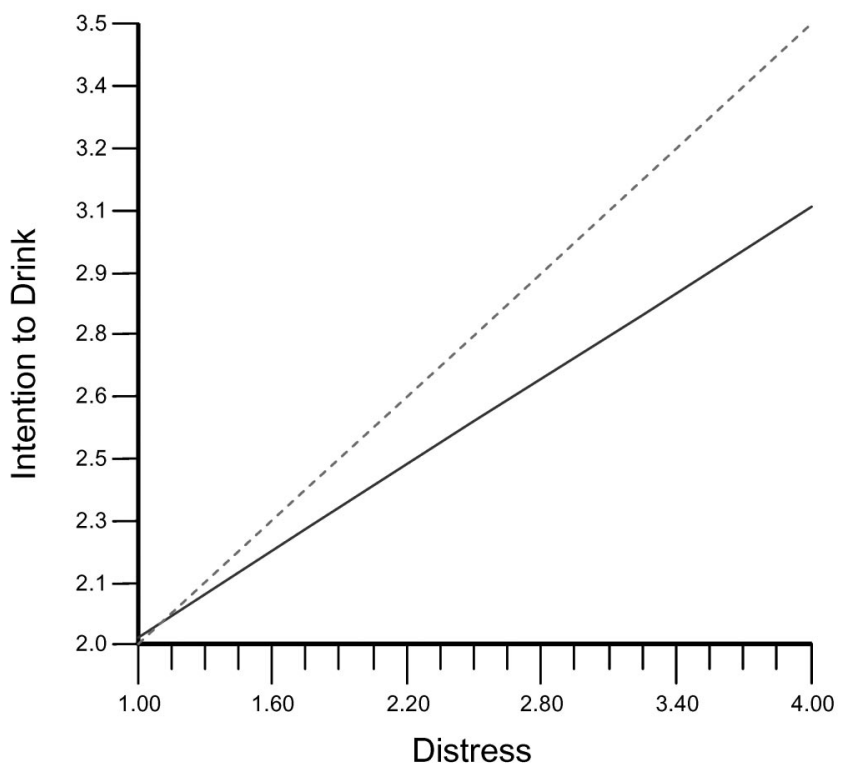

Figure 4. Relationship between self-reported distress in the morning and intention to drink that day, controlling for day of week and previous day's alcohol consumption, in Study 2. Lines are plotted at one standard deviation above and below the sample mean of average weekly drinking. Stippled line $=$ heavy drinker; solid line $=$ light drinker .

stronger the violation of their drinking limit, the more they were distressed by their alcohol consumption the following day. The relationship between distress and limit violation was above and beyond the effects of amount of alcohol consumed and hangover symptoms, which suggests that distress is related to violating a personal limit rather than just a response to acute symptoms or caused by excessive alcohol consumption.

Reciprocally, individuals' morning state was related to later consumption. Experiencing distress over alcohol consumption was associated with poorer regulation of alcohol intake in both samples of social drinkers. There was a direct relationship from distress over a limit violation to the consumption of alcohol that day, as well as violating personal drinking limits later that day, in the first sample of underage social drinkers. Distress was even related to drinking the following day. The adult social drinkers in Study 2 who felt more distress showed greater intent to drink that night. Those who intended to drink that day in fact consumed more alcohol that day. Thus, in Study 2 the effect of distress on subsequent alcohol consumption was mediated by intention to drink.

The failure to find a direct effect from distress over alcohol consumption to amount consumed in Study 2 may have been caused by a smaller sample or some other mediator that was not measured, which suppressed the direct effect of distress on drinking. Indeed, research on tests of mediation has suggested that for small samples, when the effects are not proximal, there need not be a direct relationship between the two variables (Shrout \& Bolger, 2002). Overall, however, individuals who feel distressed about their previous day's alcohol consumption appear to consume more alcohol later that day than individuals who do not feel as distressed.

These two studies are consistent with models of emotional distress leading to poorer self-control (e.g., Collins \& Lapp, 1991;
Marlatt \& Gordon, 1985; Tice et al., 2001). The findings replicate and extend, in an ecologically valid manner, previous questionnaire and laboratory research (Bensley et al., 1990; Collins \& Lapp, 1991; Collins et al., 1994) that found that violating a personal limit on alcohol intake leads to guilt and remorse. In particular, negative affect after a limit violation was found to completely mediate the relationship between limit violation and subsequent drinking. Although it is impossible to establish causation in a correlational design, the temporal patterns observed (distress over drinking was measured separately from both the limit violation and subsequent drinking in Study 1) and the mediational role of emotional distress strongly suggest that the LVE process is operating.

In addition to demonstrating the negative effect of distress over drinking on the regulation of alcohol intake, the results may shed some light on how responses to negative emotions can contribute to misregulation over time and lead to trait differences in selfcontrol behavior. In particular, an examination of the moderating influences of individual differences found that the LVE process is stronger for heavier drinkers as compared to light drinkers. Heavy drinkers are more sensitive to limit violations (by responding with greater distress) and are more responsive to that distress (by drinking more). That is, the slopes are steeper for heavier drinkers than for light drinkers, which could help potentiate a downward spiral. This is true for both the underage drinkers (see Study 1) and the community sample (see Study 2). Although no participant in either study can be considered a problem drinker, the results at least are suggestive that distress hits heavier drinkers harder than light drinkers, which is consistent with the LVE model.

Other individual differences also moderate the process of the LVE. Most significantly, trait restriction, or individuals' typical desire to hew to their self-imposed limits (Collins et al., 2000; Collins \& Lapp, 1992), moderated the relationship between violating a limit and distress. Individuals who are more concerned about their limits respond to a violation of those limits with greater distress. This is consistent with previous research that also has found that restriction increases self-blame after a violation of a self-imposed limit (Collins et al., 1994; Palfai, 2001). Other individual differences also played a role in the LVE. Gender was a moderator, so that men were more sensitive to limit violations than women and responded with more distress. However, the influence of gender on the relationship between guilt and subsequent drinking was found only in Study 1.

Our findings have clinical implications for disrupting relationships that contribute to excessive drinking by some social drinkers. The teaching of behavioral skills for regulating alcohol use likely will lessen the occurrence of limit violations. If violations do occur, then intervening to change initial negative affective reactions to excessive drinking may lessen the likelihood that individuals will continue to drink to repair their negative mood, alleviate their distress, or both. For example, it may be useful to teach nondrinking-related strategies (e.g., engaging in favorite positive activities or seeking out positive social contacts) and coping skills for handling negative moods. In addition, to the extent that selfblaming attributions contribute to experiences of the LVE and related subsequent drinking, then cognitive interventions that encourage alternative situational and external attributions are likely to lessen self-blame and related distress, thereby reducing drinking (Beck, Wright, Newman, \& Liese, 1993; Marlatt, 1985). Our 
individual-difference data suggest that such interventions may be particularly helpful for heavy drinkers, persons concerned about maintaining limits on their alcohol intake, and men.

The present research complements the self-medication model that has found that individuals sometimes cope with negative emotions and unpleasant situations by increasing their alcohol intake (e.g., Armeli, Carney, Tennen, Affleck, \& O'Neil, 2000; Carney, Armeli, Tennen, Affleck, \& O'Neil, 2000; Swendsen et al., 2000). Previous research has not focused on distress over drinking, however, and has not examined how previous drinking affects individuals' subsequent moods. Thus, the present research adds to knowledge because it examined a process that occurs over time and is a strong test of the predictions made by the LVE model. This research also helps to illuminate the processes that underlie the negative effects of emotions on self-control (Tice et al., 2001).

One of the strengths of the present research is the within-subject, ecologically valid, longitudinal design provided by EMA methods. By following participants as they conducted their lives, we got a better sense of how much they drink and their responses to drinking, something that would be difficult or impossible to do in a laboratory setting. For example, in Study 1, limit violations were reported immediately after drinking; distress related to drinking was reported the next morning; and subsequent alcohol consumption was reported later in the day, as it happened. However, because of the correlational nature of the studies, strong statements of causality are not possible. The data were collected prospectively, and therefore these studies avoided many of the biases associated with self-report (e.g., memory failures and certain demand characteristics) and could suggest causality (Shiffman \& Stone, 1998). Indeed, there is reason to believe that EMA methodology leads to more accurate and honest responding and similar reactivity compared with other methods of self-monitoring (Collins \& Muraven, in press; Hufford, Shields, Shiffman, Paty, \& Balabanis, 2002; Litt, Cooney, \& Morse, 1998).

The present studies also had the advantage of using two distinct samples. The samples differed greatly in their drinking history, developmental stage, legal status, and age, yet the basic LVE process was remarkably similar in both samples. Taken together, the samples consisted of almost 150 participants who provided more than 2,500 observations. These data provided a dynamic and rich insight into a diverse group of social drinkers as they went about their daily lives.

Our studies also possessed some limitations. In particular, participants' attributions for their limit violation were not measured. Future research should consider testing for the role of attributions, as the LVE model suggests that individuals who make internal attributions for their limit violation (i.e., self-blame) may be at greater risk for increased consumption as compared to individuals who make external attributions. The use of other populations, such as problem drinkers, to better investigate the role of the LVE in the progression from alcohol use to misuse may be warranted. Participants' alcohol consumption and other variables were self-reported (albeit not retrospectively) and therefore may be subject to biases. Finally, the present research looked at the effect of distress on the self-regulation of alcohol intake, with a particular focus on guilt over violating a self-imposed limit. Investigators may want to differentiate between shame and guilt in response to a limit violation, as these are two distinct experiences and may lead to different outcomes (Tangney \& Dearing, 2002).
These studies provide evidence that distress over violating a personal limit on alcohol consumption is damaging to self-control. This is consistent with the LVE model of drinking regulation. The morning after drinking, if social drinkers consumed more than they intended, guilt is a likely response. This distress appears to increase the likelihood of drinking that day, which in turn results in more drinking, potentially creating a negative-feedback loop. Thus, through a complex series of attributions and emotions, one day's drinking can lead to the failure to regulate subsequent day's drinking, possibly setting into motion a process that could increase the individual's vulnerability to drinking problems.

\section{References}

Argeriou, M. (1975). Daily alcohol consumption patterns in Boston: Some findings and a partial test of the Tuesday hypothesis. Journal of Studies on Alcohol, 36, 1578-1583.

Armeli, S., Carney, M. A., Tennen, H., Affleck, G., \& O’Neil, T. P. (2000). Stress and alcohol use: A daily process examination of the stressorvulnerability model. Journal of Personality and Social Psychology, 78, 979-994.

Baron, R. M., \& Kenny, D. A. (1986). The moderator-mediator variable distinction in social psychology research: Conceptual, strategic, and statistical considerations. Journal of Personality and Social Psychology, 51, 1173-1182.

Baumeister, R. F., Heatherton, T. F., \& Tice, D. M. (1994). Losing control: How and why people fail at self-regulation. San Diego, CA: Academic Press.

Beck, A. T., Wright, F. D., Newman, C. F., \& Liese, B. S. (1993) Cognitive therapy of substance abuse. New York: Guilford Press.

Bensley, L. S., Kuna, P. H., \& Steele, C. M. (1990). The role of drinking restraint success in subsequent alcohol consumption. Addictive Behaviors, 15, 491-496.

Bryk, A. S., Raudenbush, S. W., \& Congdon, R. T. (2000). HLM (Version 5.0). Chicago: Scientific Software International.

Carney, M. A., Armeli, S., Tennen, H., Affleck, G., \& O’Neil, T. P. (2000). Positive and negative daily events, perceived stress, and alcohol use: A diary study. Journal of Consulting and Clinical Psychology, 68, $788-$ 798.

Collins, R. L. (1993). Drinking restraint and risk for alcohol abuse. Experimental and Clinical Psychopharmacology, 1, 44-54.

Collins, R. L., Koutsky, J. R., \& Izzo, C. V. (2000). Temptation, restriction, and the regulation of alcohol intake: Validity and utility of the Temptation and Restraint Inventory. Journal of Studies on Alcohol, 61, 766773

Collins, R. L., Koutsky, J. R., Morsheimer, E. T., \& MacLean, M. G. (2001). Binge drinking among underage college students: A test of a restraint-based conceptualization of risk for alcohol abuse. Psychology of Addictive Behaviors, 15, 333-340.

Collins, R. L., \& Lapp, W. M. (1991). Restraint and attributions: Evidence of the abstinence violation effect in alcohol consumption. Cognitive Therapy and Research, 15, 69-84.

Collins, R. L., \& Lapp, W. M. (1992). The Temptation and Restraint Inventory for measuring drinking restraint. British Journal of Addiction, 87, 625-633.

Collins, R. L., Lapp, W. M., \& Izzo, C. V. (1994). Affective and behavioral reactions to the violation of limits on alcohol consumption. Journal of Studies on Alcohol, 55, 475-486.

Collins, R. L., Morsheimer, E. T., Shiffman, S., Paty, J. A., Gnys, M., \& Papandonatos, G. (1998). Ecological momentary assessment in a behavioral drinking moderation training program. Experimental and Clinical Psychopharmacology, 6, 306-315.

Collins, R. L., \& Muraven, M. (in press). EMA assessment for alcohol 
consumption. In A. A. Stone, S. Shiffman, A. A. Atienza, \& L. Nebeling (Eds.), The science of real-time data capture: Self-report in health research. New York: Oxford University Press.

Cooper, M. L., Frone, M. R., Russell, M., \& Mudar, P. (1995). Drinking to regulate positive and negative emotions: A motivational model of alcohol use. Journal of Personality and Social Psychology, 69, 990-1005.

Greenfield, T. K., Guydish, J., \& Temple, M. T. (1989). Reasons students give for limiting drinking: A factor analysis with implications for research and practice. Journal of Studies on Alcohol, 50, 108-115.

Heatherton, T. F., Polivy, J., \& Herman, C. P. (1990). Dietary restraint: Some current findings and speculations. Psychology of Addictive Behaviors, 4, 100-106.

Hufford, M. R., Shields, A. L., Shiffman, S., Paty, J., \& Balabanis, M. (2002). Reactivity to ecological momentary assessment: An example using undergraduate problem drinkers. Psychology of Addictive Behaviors, 16, 205-211.

Kenny, D. A., Kashy, D. A., \& Bolger, N. (1998). Data analysis in social psychology. In D. T. Gilbert, S. T. Fiske, \& G. Lindzey (Eds.), Handbook of social psychology (4th ed., Vol. 1, pp. 233-265). New York: McGraw-Hill.

Kenny, D. A., Korchmaros, J. D., \& Bolger, N. (2003). Lower level mediation in multilevel models. Psychological Methods, 8, 115-128.

Kreft, I. G. G., de Leeuw, J., \& Aiken, L. S. (1995). The effects of different forms of centering in hierarchical linear models. Multivariate Behavioral Research, 30, 1-21.

Krull, J. L., \& MacKinnon, D. P. (1999). Multilevel mediation modeling in group-based intervention studies. Evaluation Review, 23, 418-444.

Litt, M. D., Cooney, N. L., \& Morse, P. (1998). Ecological momentary assessment (EMA) with treated alcoholics: Methodological problems and potential solutions. Health Psychology, 17, 48-52.

Marlatt, G. A. (1985). Relapse prevention: Theoretical rationale and overview of the model. In G. A. Marlatt \& J. R. Gordon (Eds.), Relapse prevention (pp. 3-70). New York: Guilford Press.

Marlatt, G. A., \& Gordon, J. R. (1980). Determinates of relapse: Implications for the maintenance of behavior change. In P. O. Davidson \& M. S. Davidson (Eds.), Behavioral medicine: Changing health lifestyles (pp. 410-452). New York: Brunner/Mazel.

Marlatt, G. A., \& Gordon, J. R. (1985). Relapse prevention. New York: Guilford Press.

Muraven, M., Collins, R. L., Morsheimer, E. T., Shiffman, S., \& Paty, J. A. (2005). One too many: Predicting future alcohol consumption following heavy drinking. Experimental and Clinical Psychopharmacology, 13, $127-136$.
Muraven, M., Collins, R. L., \& Nienhaus, K. (2002). Self-control and alcohol restraint: An initial application of the self-control strength model. Psychology of Addictive Behaviors, 16, 113-120.

Norcross, J. C., Ratzin, A. C., \& Payne, D. (1989). Ringing in the new year: The change processes and reported outcomes of resolutions. Addictive Behaviors, 14, 205-212.

Palfai, T. P. (2001). Individual differences in temptation and responses to alcohol cues. Journal of Studies on Alcohol, 62, 657-666.

Polivy, J., \& Herman, C. P. (1999). The effects of resolving to diet on restrained and unrestrained eaters: The "false hope syndrome." International Journal of Eating Disorders, 26, 434-447.

Ruderman, A. J., \& McKirnan, D. J. (1984). The development of a Restrained Drinking Scale: A test of the abstinence violation effect among alcohol users. Addictive Behaviors, 9, 365-371.

Schwartz, J. E., \& Stone, A. A. (1998). Strategies for analyzing ecological momentary assessment data. Health Psychology, 17, 6-16.

Selzer, M. L., Vinokur, A., \& Rooijen, L. (1975). A self-administered short Michigan Alcohol Screening Test (SMAST). Journal of Studies on Alcohol, 36, 117-126.

Shiffman, S., Paty, J. A., Gnys, M., Kassel, J. A., \& Hickcox, M. (1996). First lapses to smoking: Within-subjects analysis of real-time reports. Journal of Consulting and Clinical Psychology, 64, 366-379.

Shiffman, S., \& Stone, A. A. (1998). Introduction to the special section: Ecological momentary assessment in health psychology. Health Psychology, 17, 3-5.

Shrout, P. E., \& Bolger, N. (2002). Mediation in experimental and nonexperimental studies: New procedures and recommendations. Psychological Methods, 7, 422-445.

Swendsen, J. D., Tennen, H., Carney, M. A., Affleck, G., Willard, A., \& Hromi, A. (2000). Mood and alcohol consumption: An experience sampling test of the self-medication hypothesis. Journal of Abnormal Psychology, 109, 198-204.

Tangney, J. P., \& Dearing, R. L. (2002). Shame and guilt. New York: Guilford Press.

Tice, D. M., Bratslavsky, E., \& Baumeister, R. F. (2001). Emotional distress regulation takes precedence over impulse control: If you feel bad, do it! Journal of Personality and Social Psychology, 80, 53-67.

West, S. G., \& Hepworth, J. T. (1991). Statistical issues in the study of temporal data: Daily experiences. Journal of Personality, 69, 609-662.

Received April 26, 2004

Revision received July 23, 2004

Accepted July 26, 2004 